\title{
Bildung und Marktregime
}

\author{
Daniel Straß
}

Online publiziert: 7. März 2015

(C) Die Autor(en) 2015. Dieser Artikel ist auf Springerlink.com mit Open Access verfügbar.

\section{Rezension zu:}

Widerspruch 63 (2013). Beiträge zu sozialistischer Politik: Bildung und Marktregime. Rotpunktverlag, Zürich, 224 Seiten, 18,00 €, ISBN 978-3-85869563-5

Die Widerspruch-Ausgabe (2/2013) verbindet unter dem Titel Bildung und Marktregime verschiedene Beiträge zur Analyse und Kritik von Bildung unter gegenwärtigen neoliberalen ,postdemokratischen“ Bedingungen. Als in gewisser Weise repräsentative Klammer um etliche der Beiträge kann ein Zitat im Anschluss an Paolo Freire im Artikel der Diskussionsgruppe Autonome Schule Zürich (ASZ) unter dem Titel Bildung zur Selbstbestimmung gelten: „Unser Ausgangspunkt ist: Es gibt keine politisch neutrale Bildung. Entweder dient Bildung der Befreiung des Menschen, oder sie behindert die Befreiung und begünstigt Unterdrückung“ (S. 103). Die klassische Bemerkung hat in mindestens dreifacher Hinsicht systematischen Wert für die Zusammenfassung der Ausgabe.

Erstens: Wenn es Neutralität in der (politischen) Bildung nicht gibt, wird sich eine kritische Perspektive schwer tun mit der bloßen Deskription bestimmter Entwicklungen im gegenwärtigen Aus- und Weiterbildungsbereich. Auch Beschreibung und Analyse empirischer Daten (Trends) geschehen dann schon mit kritischer Intention bzw. Diktion, wie es sich auch in den eher deskriptiven Beiträgen zur Bildungspolitik (Exzellenz statt Chancengerechtigkeit?, S. 18 ff.) und zur berufsorientierten Bildung (S. 27 ff.) in der Schweiz zeigt, die in den Augen von Karl Weber weiterhin eine hierarchische Schichtung in den Bildungsniveaus reproduziert und von der Leitvorstellung der Berufsförmigkeit der Qualifikationen geprägt ist, also weniger von einer „organisationsbezogenen“ Allgemeinbildung. Auch Aspekte von Bildungsverläufen

Dr. D. Straß $(\bowtie)$

Halle, Deutschland

E-Mail: daniel.strass@paedagogik.uni-halle.de 
wie Brückenangebote zwischen der obligatorischen und nachobligatorischen Bildung in der Schweiz (S. 53 ff.) werden noch in ihrer empirischen Darstellung als „Problemverschleierung" dechiffriert und zwar insofern als Sibylle Künzli und Regina Scherrer zeigen können, dass ,[...] Brückenangebote mithelfen, Mechanismen des obligatorischen Bildungssystems, beispielsweise die Problematik der frühen Selektion, zu verschleiern: Strukturelle Mängel werden zu individuellem Versagen“ (S. 60).

Mit der nicht-neutralen Bildung liegt zweitens eine systematische Reflexion auf das Spannungsfeld Bildung - Wissenschaft nahe, insofern die Bildung eine gewisse Wertorientierung wie gezeigt häufig inhärent hat und die Wissenschaft idealtypisch wertfrei ist. Sie soll also etwas sein, was oben für (politische) Bildung ausgeschlossen wurde. Diese Problematik berührt explizit der sehr lesenswerte Artikel Wertfrei und neutral? (S. 189 ff.) von Ueli Mäder und Hector Schmassmann. Implizit ist das Spannungsfeld auch präsent im Artikel Geschlechterperspektiven in der , unternehmerischen Hochschule ' (S. 77 ff.), die die gegenwärtige Verbetriebswirtschaftlichung des Hochschulsystems als neuen Verhinderer von Anliegen der Gender Studies entdeckt, insofern hinter der outputorientierten Verwertbarkeitslogik von forschungsrelevanten Themen häufig dennoch hintergründige Klientelgruppen beobachtet werden, was die vermeintliche Wertfreiheit von Wissenschaft als opak erscheinen lässt, - allerdings unfreiwillig auch im Hinblick auf die „Gender Studies“ selbst, von denen zugegeben wird, dass ihre programmatische Inklusion in den Wissenschaftsbetrieb vielfach einer „Nötigung“ (S. 79) entsprach.

Drittens: Wo der Nicht-Ort der Neutralität zugunsten eines profilierten Verständnisses emanzipatorischer, kritischer Bildung überschritten wird, wie im Eröffnungsbeitrag von Ulrich Brand (Demokratie und emanzipatorische globale Bildung, S. 7 ff.) wird ein Plädoyer für ein ,kritisch-weltgesellschaftliches Bildungsverständnis“"gehalten. Der Beitrag lotet die analytischen Vorteile des Begriffs des Postneoliberalismus aus, in dessen Kielwasser nicht nur systemimmanente Veränderungen eines sich selbst erodierenden Neoliberalismus, sondern auch weltweite kritische vernetzte Initiativen (z. B. globalisierungskritische Proteste) thematisiert werden können. Damit will er die Reflexion auf wirkliche Demokratisierung als „Lebenspraxis und Begehren, die sich jenseits der liberalen Demokratie entwickelt" (S. 12), und ein damit verbundenes emanzipatorisches Bildungsverständnis mit Reflexion herrschaftsstabilisierender Mechanismen von Bildung vorantreiben. Trotz der luziden Beobachtungen zeigt sich aber auch, dass die Möglichkeiten des Begründungsraumes praktischer und politischer Philosophie nicht voll ausgeschöpft werden. Letztlich ist eine Kritik an gegenwärtiger Bildung, also einer ökonomisierten Bildung als „Wasserträger des Systems“ (Pongratz), wirkungsvoll nur vor dem Hintergrund einer Reflexion auf die Idee der eudaimonia, des guten, gelingenden Lebens möglich. Wer kritisiert, hat eine Ahnung von etwas, das er selbst nicht der demokratischen Willensbildung aussetzen würde, weil das Legitimation auf Zeit und damit Austauschbarkeit bedeutete.

In der Summe handelt es sich um lesenswerte Beiträge, deren Potential im Hinblick auf das ,ewige Lamento ' hinsichtlich einer „Ökonomisierung der Bildung“ aber in Richtung philosophischer Begründungen für Alternativen jenseits bloß immanenter Kritik hätte überstiegen werden können. 
Open Access Dieser Artikel unterliegt den Bedingungen der Creative Commons Attribution License. Dadurch sind die Nutzung, Verteilung, und Reproduktion erlaubt, sofern der/die Originalautor/en und die Quelle angegeben sind. 